\title{
LA PRODUCCIÓN Y LA COMPRENSIÓN DE LA CIENCIA: LA ELABORACION EN EL APRENDIZAJE DE LA CIENCIA ESCOLAR
}

OTERO, J.

Departamento de Física. Universidad de Alcalá. Madrid.

\section{SUMMARY}

This paper criticizes the conceptual reformulations of science made with pedagogical purposes and, more particularly, the absence of reference to originary problems.

\section{INTRODUCCIÓN}

La mejora del aprendizaje de la ciencia exige actuaciones diversas. Los problemas didácticos que se planteaban hace 10 ó 15 años en nuestro país; por ejemplo, se relacionaban fundamentalmente con la mejora de los medios y las técnicas de enseñanza. En una revisión parcial de la literatura sobre didáctica de la física aparecida en 8 revistas educativas (1) en el periodo 1970-1980 (recuérdese que "Enseñanza de Ias Ciencias" aparece en 1983) encontramos que el $50 \%$ de los artículos estaba dedicado a métodos y medios de enseñanza (Otero y Velarde 1980). Eran muy escasos los artículos en estas revistas cuya orientación fundamental fuese, p.e. la aplicación de teorías psicológicas (por ejemplo, del aprendizaje) a Ia enseñanza de la física. EI panorama ha cambiado desde entonces. Desde hace años se viene prestando atención, a la forma en que el alumno aprende, en especial a lo que ya sabe como determinante de lo que es capaz de aprender. Sin embargo hay otras variables que deben merecer también la atención de los investigadores en didáctica de la ciencias. Una de ellas es la naturaleza del conocimiento científico que se enseña. En el año 1964, J. Schwab denunciaba la poca atención que se había prestado a la materia de enseñanza, uno de los cuatro eiementos que concibe como componentes de la educación, junto con el alumno, el profesor y el medio en que tiene lugar el aprendizaje:

"Solamente la materia de ensefianza, entre los cuatro, se ha relegado a la posición de una buena esposa: se tiene por conocida, no cambia y está siempre dispuesta para lo que se necesite". (Schwab 1964, p. 4).

La observación describe bastante bien la situación para la enseñanza de Ias ciencias en los niveles medio e inicios del superior, a los cuales se refiere este artículo. Es muy posible que los métodos, por ejemplo, hayan variado en las últimas décađas. También las ideas sobre la forma en que el alumno aprende. Sin embargo, la estructura conceptual de la ciencia que se enseña en estos cursos se considera con mucha frecuencia como algo dado e inamovible. Ha estado normalmente fuera del alcance e interés de la investigación educativa: no constituye una variable problemática en las reflexiones y estudios sobre didáctica de las ciencias. Las formulaciones de la ciencia que se presenta a los alumnos, parecen inmutables y las únicas posibles. Pero, ¿por qué el conocimiento científico se presenta de la manera que se hace? ¿Son esas estructuras conceptuales las únicas posibles? Este artículo se refiere a problemas de aprendizaje que plantean las estructuras conceptuales tradicionales de la ciencia escolar, y que tienen su raíz en la naturaleza de la llamada "ciencia pública".

\section{LA CIENCIA PRIVADA Y LA CIENCIA PÚBLICA}

Tomemos, por ejemplo, una presentación frecuente dei concepto de carga eléctrica en los libros de texto de física del nivel de bachillerato:

"La electrización de cualquier cuerpo se puede poner de manifiesto mediante un péndulo eléctrico, que consiste en un hilo de seda que termina en una esferita de médula de sauco. Si aproximamos al péndulo un trozo de vidrio previamente frotado con un paño, atraerá a la esferita y tan pronto como la toque será repelida; si acercamos un trozo de resina, también frotada previa- 
mente, atraerá enérgicamente a la bolita. Si en vez de iniciar el experimento con el vidrio lo empezamos con la resina, ésta atraerá a la esferita para repelerla enseguida, lo mismo que antes el vidrio. Si después de haber tocado la bolita con la resina, se le acerca el vidrio, será fuertemente atraída".

Estas experiencias quedan perfectamente aclaradas si se establecen dos clases de electricidad: la vítrea o positiva y la resinosa o negativa. Dos cuerpos cargados con electricidad del mismo signo se repelen y de signos contrarios se atraen.

¿Cuál es la razón por la que el concepto de carga positiva y negativa deba ser presentada así? ¿Se han inventado los conceptos de carga positiva y negativa simultáneamente a la observación de fenómenos como los que se indican? La respuesta está relacionada con Ia diferencia entre las formas en que se produce y se presenta la ciencia.

En el estudio de la naturaleza de la ciencia se ha distinguido entre "contexto de descubrimiento" y "contexto de justificación" (Reichenbach 1938), o entre "ciencia privada" y "ciencia pública" (Holton 1973). La idea que subyace a estas nociones es la distinción entre la ciencia en desarrollo, es decir, el proceso de creación de las ideas científicas, y la ciencia hecha, lista para ser transmitida a la comunidad científica. Los problemas que plantea el estudio de una u otra faceta de la ciencia son diferentes. Popper (1959), por ejemplo, considera que la psicología dei conocimiento debe ocuparse de lo que plantea la ciencia en desarrollo mientras que la lógica del conocimiento científico se ocupa de la justificación de la validez de la ciencia tal como se presenta después del proceso de creación:

"El problema de cómo es que se le ocurren nuevas ideas a un hombre - sea un tema musical, un drama o una teoría científica- puede ser de gran interés para la psicología empírica: pero es irrelevante para el análisis lógico del conocimiento científico. Este último no se ocupa de cuestiones de hecho (el, quid facti? de Kant), sino solamente de cuestiones de justificación de la validez (el quid juris? de Kant). Sus preguntas son de la clase siguiente: ¿puede justificarse un enunciado? Si es así ¿cómo?... De acuerđo con esto, distinguiré claramente entre el proceso de concepción de una nueva idea, y los métodos y resultados de examinarla lógicamente". (Popper, op. cit., p. 31).

El paso de una a otra clase de ciencia no está exento de problemas. Medawar (1963) advertía sobre los peligros del tránsiło de la ciencia en desarrollo a la ciencia pública representada por el artículo científico típico. Este último da, en su opinión, una imagen frauđulenta de la actividad de producción científica, es decir, de la ciencia privada:

"Volviendo de nuevo al artículo científico: el artículo científico es un fraude en el sentido de que verdaderamente presenta una narración totalmente equivocada de Ios procesos de pensamiento implicados en los descubrimientos científicos." (p. 378).
La cuestion que interesa resaltar aquí es que la ciencia pública, que se presenta a los estudiantes, al igual que la que se materializa en un artículo, resulta de una reformulación de la ciencia privada desde el contexto de descubrimiento. La presentación del concepto de carga eléctrica, al que nos referíamos antes, a partir de los datos de la experiencia, es un ejemplo de esta reformulación. Al concepto de carga eléctrica positiva y negativa no se llegó directamente a partir de la observación de fenómenos de atracción y repulsión. Resultó de un largo proceso evolutivo en donde los fenómenos que hoy parecen servir de apoyo a lo que se presenta como única interpretación conceptual fueron interpretados de muy diversas maneras. Gilbert, precursor de los investigadores sobre la electricidad y el magnetismo, observó en el siglo XVI los fenómenos de repulsión eléctrica, por ejemplo, sin concederles atención especial: los atribuía a un agotamiento del efluvio que emitían los cuerpos electrizados y que era responsable de la atracción que ejercían sobre otros cuerpos. Cabeo en el siglo XVII interpretaba esta misma repulsión como un fenómeno puramente mecánico de rebote: el cuerpo electrizado al emitir el "efluvio eléctrico" desplaza el aire en sus proximidades; este aire desplazado puede formar remolinos al entrar en contacto con el aire en reposo; los remolinos pueden arrastrar partículas ligeras en las proximidades del cuerpo electrizado de tal manera que al chocar violentamente con éste, rebotan. No hay necesidad, por tanto, de postular la existecia de dos tipos de efluvios eléctricos. Esta hipóstesis, de hecho, mereció la suficiente atención como para pro. mover la realización de trabajos experimentales (RoIIer y Roller 1970, p. 14). Franklin en el siglo XVIII interpretaba los mismos datos experimentales que presenta el libro de texto, en términos de un único tipo de carga eléctrica con propiedades atractivas. La repulsión se explicaba suponiendo que las partículas de materia se repelen entre sí; la ausencia de fluido eléctrico rompería el equilibrio favoreciendo la fuerza repulsiva.

Un largo proceso histórico de creación, como el anterior, se sintetiza de tal manera que los resultados (los conceptos de cara $+y-$ ) se presentan como si se relacionasen unívocamente con estos hechos. Sin embargo, Ia relación presentada entre hechos e interpretación conceptual distaba de ser evidente para muchas mentes geniales a lo largo de la historia.

Otro ejemplo de las reformulaciones resultantes deI tránsito de la ciencia privađa a la ciencia pública, puede encontrarse en la presentación de la segunda ley de la termodinámica hecha por muchos textos:

"Aunque el rendimiento de las máquinas térmicas ha aumentado de manera considerable desde las primeras máquinas de vapor, es imposible construir una máquina térmica que alcance el $100 \%$ de rendimiento, es decir, que no ceda calor a un foco que se encuentre a una temperatura más baja. Este resultado experimental es conocido como el enunciado de Kelvin-Planck del segundo principio de la termodinámica: Es imposible para una máquina que trabaje cíclicamente extraer calor de un foco y realizar una cantidad equivalente de 


\section{INVESTIGACIÓN Y EXPERIENCIAS DIDÁCTICAS}

trabajo, sin producir otro efecto. (Tipler 1976, p. 465).

El enunciado de Kelvin-Planck se postula presentándolo como "resultado experimental" o como "una generalización de la experiencia" (Halliday Resnick 1977, p. 564). Kelvin, sin embargo, no introdujo la segunda ley generalizando a partir de la experiencia sino que la enunció como un principio que era necesario para demostrar el teorema de Carnot, una vez abandonada la teoría del calórico sobre la cual había sustentado Car. not la demostración:

"...Debe admitirse que la demostración original de Car. not falla claramente, pero no podemos inferir que la proposición a la que se ha llegado (el teorema de Carnot) sea falsa... Hasta el principio de este año no pude encontrar la demostración que se da más arriba, por medio de la cual se establece la verdad de la proposición sobre la base de un axioma (el enunciado de Kelvin de la segunda ley) que creo que puede ser admitido por todo el mundo..." (W. Thomson 1882, p. 181).

Sin embargo el enunciado de Kelvin-Planck no se presenta de esta manera, es decir, como fundamentación necesaria para la demostración del teorema de Carnot cuya verdad se admite previamente. Se suele considerar más conveniente introducirlo directamente, relacionándolo con la imposibilidad de que existan máquinas térmicas con un rendimiento del $100 \%$ "una generalización de la experiencia".

Los ejemplos anteriores intentan poner de manifiesto que la estructura de la ciencia pública no es obvia o inmutable, sino que es el resultado de una reformulación de la ciencia privada. ¿Cuáles son, entonces, las razones que guían esta transformación? Para la enseñanza parecería sensato que primasen las razones pedagógicas: la ciencia así reformulada debería ser más fácil de aprender. Interesa, por tanto, examinar desde el punto de vista del aprendizaje una de las características de esta reformulación (2): la ausencia de problemas en las reformulaciones tradicionales del contenido con. ceptual de la ciencia utilizadas con propósitos pedagógicos.

\section{LAS ESTRUCTURAS CONCEPTUALES TRADICIONALES: "UN DISCURSO CEN" TRADO EN LAS CONCLUSIONES"}

Una gran parte de la ciencia escolar consiste en un conjunto de herramientas conceptuales que se proporcio. nan al alumno, aparentemente salidas de la nada, y cuya justificación se encontraría siempre a posteriori: lo que Schwab (1964) llamó hace tiempo, "discurso centrado en las conclusiones", y que presentaba como argumento para enseñar la ciencia mediante un proceso de investigación (3). En particular, el contenido conceptual de la ciencia que se presenta al alumno no contiene problemas sino únicamente las soluciones. ¿Qué problema, por ejemplo, vino a solucionar el concepto de carga eléctrica o la segunda ley de la termodinámica? ¿Qué pregunta se está respondiendo? Tomemos otro ejem- plo: en la formulación del teorema de Camot es el máximo que se puede obtener entre dos temperaturas y que es independiente de la sustancia de trabajo. Carnot y otros investigadores del siglo XVIII, condicionados por las exigencias de la Revolución Industrial, tenían claro el problema y la pregunta que intentaban contestar. En las primeras páginas de "Réflexions sur la puissance motrice du feu", la obra en que Carnot hace sus aportaciones fundamentales, explica:

"Se ha planteado frecuentemente la pregunta de si el poder motriz del calor es limitado o no tiene límites; de si las posibles mejoras de las máquinas térmicas tienen un límite asignable, límite que la propia naturaleza de las cosas impida sobrepasar de manera alguna, o si, al contrario, estos perfeccionamientos pueden llevarse a cabo indefinidamente. Se ha buscado durante mucho tiempo, y se busca todavía hoy, si no existirían agentes preferibles al vapor de agua para desarrollar el poder motriz del calor; si a este respecto no presentaría el aire atmosférico, por ejemplo, mayores ventajas. Nos proponemos someter estas cuestiones a un examen cuidadoso." (Carnot 1824, p. 6).

Carnot buscaba (y encontró) la respuesta. Los alumnos actuaies tienen ante sí claramente la respuesta:

El rendimiento es el mismo para todas las máquinas reversibles que trabajen entre los mismos dos focos caloríficos, sea cual fuere la sustancia de trabajo: gas ideal, gas real o agua. (Tipler 1976, p. 473).

Deben buscar, sin embargo, la pregunta que se está contestando; no está incluida en los contenidos tradicionales de la ciencia de estos niveles.

La supresión del problema es consecuencia de la reformulación y el tránsito de la ciencia privada a la ciencia pública. Thomas Kuhn en "La estructura de las revolusiones científicas" (1969) hace algunas consideraciones sobre las características de la educación científica, la formación en lo que llama "ciencia normal", que arrojan alguna luz sobre las características de las reformulaciones pedagógicas del conocimiento científico. El propósito de la educación científica es formar a los futuros científicos en el manejo y conocimiento del paradigma dominante. Esta formación no incluye la reflexión sobre este paradigma:

"Aunque muchos científicos hablan fácilmente y bien sobre las hipótesis individuales particulares subyacentes a una parte concreta de la investigación actual, son poco mejores que los profanos cuando se trata de caracterizar las bases establecidas de su campo, sus problemas y métodos legítimos." (Kuhn, op. cit., p. 46).

Esta educación “... desde luego es estrecha y rígida, probablemente más que ninguna otra excepto, quizá, la teología ortodoxa. Pero para el trabajo científico normal, para la resolución de puzzles dentro de la tradición definida por los líbros de texto, el científico está equipado casi de manera perfecta" (Kuhn, op. cit., p. 166). Por tanto, quizá la reflexión y la enseñanza de las preguntas que desencadenaron las respuestas conceptuales que se presentan a los alumnos es una actividad 
superflua desde este punto de vista. El futuro científico debe dominar el paradigma dentro del que_trabaja y aplicarlo a la resolución de nuevos "puzzles". El dominio del paradigma y la reflexión sobre él son consideradas como actividades separadas. Esta es una época en que se enseña a los alumnos de ciencias fundamentalmen. te lo que Touimin (1972) llama "mirar hacia fuera":

"El problema de la comprensión humana es doble. $\mathrm{El}$ hombre conoce y es consciente al mismo tiempo de que conoce. Adquirimos, poseemos y hacemos uso del conocimiento; pero al mismo tiempo somos conscientes de nuestra propia actividad como sujetos cognitativos. Como consecuencia de ello, la comprensión humana se ha desarrollado históricamente de dos formas complementarias: ha crecido, pero al mismo tiempo se ha profundizado, conviertiéndose simultáneamente en más extensa y más reflexiva. Mirando "hacia fuera" y dominando los problemas que nos presenta el mundo en que vivimos, hemos extendido nuestra comprensión; mirando "hacia dentro" y considerando como hemos dominado esos problemas, la hemos profundizado. Y a través de la historia del pensamiento estas dos actividades han sido continuamente en paralelo... Ha habido épocas -incluyendo algunos de los períodos más fructíferos del pensamiento humano- en que estas dos actividades se veían como íntimamente relacionadas... Ha habido otras épocas -incluyendo gran parte de nuestro siglo veinte- en que las dos actividades se veían como completamente separadas e independientes..." (p. 1).

Sería lícito, por tanto, plantear la pregunta de si la educación científica descrita por Kuhn, la enseñanza para "mirar hacia fuera", es apropiada para toda la educación científica y en todos los niveles. ¿Puede haber alguna razón por la que sea inadecuado aprender solamente los resultados conceptuales de Ia ciencia, y la forma en que se aplican, ignorando componentes de la ciencia en desarrollo como los problemas que dieron lugar a las respuestas conceptuales que recogen los libros de tex to? A continuación se propone una hipótesis sobre los efectos que tienen en el aprendizaje las reformulaciones escolares tradicionales del conocimiento científico y, en particular, la supresión de problemas en estas reformulaciones.

\section{LOS PROBLEMAS DE APRENDIZAJE}

De acuerdo con la teoría del aprendizaje de Ausubel (Ausubel et. al. 1978), el aprendizaje significativo tiene lugar cuando el que aprende conecta de manera no arbitraria Ia nueva información a iđeas que ya posee. Otros investigadores en psicología del aprendizaje, han prestado atención a este proceso de conexión o elaboración de la nueva información. Stein y Bransford (1979), por ejemplo, realizaron experimentos relacionados con el tema. Leyeron a estudiantes univer. sitarios frases como: "El hombre calvo leía el periódico", "El hombre bajo usaba la escoba", "EI hombre fuerte hojeaba el libro", "El hombre alto compraba las galletas", etc. Debe notarse que no hay razón particular alguna para ligar una cierta clase de hombre con la actividad correspondiente: las conexiones son arbitrarias. Se pedía a los estudiantes que atendiesen a las frases y evaluasen lo comprensibles que eran. A continuación se realizó una prueba, no anunciada previamente, en la que se hacían preguntas como: "Qué hombre leía el periódico?". Los resultados ponían de manifiesto que los alumnos recordaban una media de 4.20 frases correctamente sobre 10. A un segundo grupo de estudiantes se le leyeron frases como: "El hombre calvo leía el periódico para buscar un anuncio de crecepelos", "El hombre bajo usaba la escoba para llamar el timbre", "El hombre fuerte hojeaba el libro sobre levantamientos de pesas", "El hombre alto compraba las galletas que estaban en el estante de arriba", etc. En este caso la actuación de los alumnos en el test posterior mejoró notablemente: recordaron una media de 7.40 frases correctamente. Stein y Bransford interpretan los resultados indicando que en el segundo caso los alumnos podían elaborar la información y entender por qué se establecía la conexión particular entre la actividad realizada y la clase de hombre, cosa que no se hacía en el primer caso en donde la conexión es arbitraria. Proporcionan también un ejemplo tomado de las ciencias en donde se da una situación similar. La proposición "Las arterias son gruesas y elásticas y las venas delgadas y poco elásticas" puede presentar a los alumnos problemas de recuerdo similares a los de la primera serie de frases. La conexión entre venas o arterias y sus propiedades puede parecer arbitraria a un principiante al que se le presente la información de esta forma. Un experto, sin embargo, ha elaborado esta información conectándola a otros conocimientos que ya posee: por ejemplo, las arterias deben ser elásticas para soportar los incrementos repentinos de presión generados por el corazón. La conexión entre arterias y elasticidad no se ve entonces como arbitraria; la proposición se recuerda mejor.

La hipótesis que se puede plantear, entonces, es que una de las dificultades para el aprendizaje significativo de las reformulaciones tradicionales del conocimiento científico se encuentra en la posibilidad de elaborar la nueva información. Al suprimir los problemas (entre otros elementos) de la reformulaciones conceptuales de la ciencia realizadas con propósitos pedagógicos, desaparece un componente que hacía menos arbitrario el contenido conceptual de la ciencia. Los conceptos científicos también "caen del cielo", como apunta Lakatos en la crítica, en ciertos aspectos paralela a la que se hace aquí, al estilo deductivo de muchas presentaciones de las matemáticas:

"Como ya se ha mencionado, el estilo deductivo arranca las definiciones generadas por las pruebas de sus "antepasados en la prueba", las presenta como caídas del cielo, de una forma artificial y autoritaria. Oculta los contraejemplos globales que llevaron a su descubrimiento. El estilo heurístico, por el contrario, resalta estos factores. Pone énfasis en la situación problema: enfatiza la "lógica" que dió lugar al nuevo concepto." (Lakatos 1977, p. 144).

Un ejemplo de las dificultades que pueden aparecer para elaborar conceptos o proposiciones presentados 
sin dar cuenta de los problemas a que estan respondien * do, se puede encontrar en la proposición, mencionada más arriba, que establece la independencia del renđimiento de una máquina de Carnot de la sustancia de trabajo. En un estudio sobre el aprendizaje de la termodinámica (Otero y Brincones 1987) se intentaba enseñar esta proposición en el contexto de una introducción a la segunda ley de la termodinámica, utilizando materiales tradicionales, a alumnos de 3을 universidad. Se encontró que un $52 \%$ de los alumnos de 30 y un $39 \%$ de alumnos de $1^{\circ}$ de universidad no la aprendieron. De hecho, el $33 \%$ de los alumnos de $3^{\circ}$ y el $33 \%$ de los de $1^{\circ}$ de universidad, nueve semanas después de haber estudiado los materiales, creían exactamente lo contrario: que el rendimiento de una máquina de Carnot funcionando entre dos temperaturas dadas depende de la utilización de una un otra sustancia de trabajo (Otero y Brincones, op. cit., p. 100). Las conexiones que se habían creado entre la proposición correcta y la estructura de conocimientos de muchos esiudiantes eran débiles: la elaboración era deficiente. En los materiales tradicionales está ausente, como se indicó anteriormente, el problema que dió lugar a la proposición: ¿Tiene algún límite el aumento del rendimiento de una máquina térmica? en particular, ¿es posible aumentar indefinidamente el rendimiento de una máquina térmica utilizando ciertas sustancias de trabajo? Por ello, los resultados pueden interpretarse de forma análoga a los obtenidos por Stein y Bransford, mencionados más arriba: es posible que la conexión entre máximo rendimiento y sustancia de trabajo sea tan arbitraria para los estudiantes a los que no se les menciona el problema de incrementar el rendimiento de las máquinas térmicas en el contex to de la Revolución Industrial, como la conexión entre hombre bajo y uso de la escoba para los sujetos a los que no se les menciona el problema de tocar un timbre demasiado alto.
Debe notarse que las dificultades de elaboración a que nos referimos están provocadas por la arbitrariedad inicial de la información científica que se presenta al alumno. Es muy probable que a través de una actividad posterior de integración, resolviendo problemas por ejemplo, el alumno consiga establecer vínculos no arbitrarios entre las proposiciones o conceptos que inicialmente veía desconectados. Sin embargo, no es la solución de los problemas iniciales de aprendizaje que se han mencionado y que sería necesario evitar.

\section{CONCLUSIONES}

Es posible que la educación en la ciencia normal, que describe Kuhn, no sea adecuada para todos los alumnos, en todas las ocasiones. Hay problemas de apredizaje que pueden tener su raíz en la estructura tradicional de la ciencia pública utilizada con propósitos pedagógicos. Se ha propuesto la hipótesis de que algunas dificuitades de elaboración del contenido conceptual de la ciencia escolar se deben a la ausencia de elementos, como los problemas, que permiten disminuir la arbitrariedad aparente que tienen para el principiante los conceptos y proposiciones de la ciencia. Ello sugiere que, para estos propósitos, sería conveniente utilizar la historia de la ciencia de forma diferente a como se ha hecho en gran parte hasta ahora. No se trata de introducir la historia como ilustración del proceso de desarrollo de la ciencia, de manera colateral a las formulaciones escolares establecidas de la ciencia pública. Se trataría más bien de usar la ciencia privada como materia prima sobre la cual trabajar para conseguir estructuras conceptuales de la ciencia escolar con más significado psicológico. El trabajo, que requeriría competencia en la materia científica, en su historia y en el proceso de aprendizaje, no parece sencillo.

\section{NOTAS}

(1) Aula Abierta, Bordón, Cuademos de Pedagogía, La Educación Hoy, Educadores, Electrónica y Física Aplicada (Separata de Didáctica), Revista de Bachillerato, Revista de Educación.

(2) Algunas de estas características se consideran en otro lugar (Otero 85).

(3) ¡El aprendizaje por descubrimiento cumple 25 años! 


\section{REFERENCIAS BIBLIOGRÁFICAS}

AUSUBEL, D., NOVAK, J. y HANESIAN, H, 1978. Educational Psychology. A Cognitive View. (Holt, Rinehart and Winston: New York). (Traducción castellana. Ed. Trillas, 1983).

CARNOT, S., 1824. Réflexions sur la puissance motrice du feu. (Chez Bachelier: Paris). Edicion crítica e introduccion de R. Fox. Paris: Librairie Phìlosophique de J. Vrin. 1978.

HALLIDAY, D. y RESNICK, R, 1977. Física. (CECSA: Méjico).

HOLTON, G., 1973. The Duality and Growth of Physical Science. En G. Holton Thematic Origins of Scientific Thought: Kepler to Einstein. (Harvard University Press: Cambridge, Mass).

KUHN, T.S., 1969. The Structure of Scientific Revolutions. (The University of Chicago Press. Chicago). (Traduccion castellana. Fondo de Cultura Económica).

LAKATOS, I., 1977. Proofs and Refutations. (Cambridge University Press: Chicago). (Traducción castellana. Alianza Editorial, 1978).

MEDAWAR, P.B., 1963. Is the Scientific Paper a Fraud? The Listener, pp. 377-378. Reimpreso en Science Teacher Education Project, Readings in Science Education. (McGraw-Hill, 1974: Londres).

OTERO, J., 1985. Assimilation problems in traditional representations of scientific knowledge, European Journal of Science Education, 7,4, p. 361-369.

OTERO, J. y BRINCONES, I., 1987. El aprendizaje significativo de la segunda ley de Ia termodinámica. Infancia y aprendizaje, 38, pp. 89-107.

OTERO, J. y VELARDE, M.G., 1980. La investigación educativa en el área de la enseñanza de la Física en el BUP y
COU. Ponencia presentada en el Simposio sobre Didáctica de la Física y la Matemática. Instituto Nacional de Ciencias de la Educación, Madrid, 25-28 de Junio de 1980.

POPPER, K.R., 1959. The Logic of Scientific Discovery. (Hutchinson: Londres), 9. reimpresión revisada, 1977. (Traducción castellana. Tecnos).

REINCHENBACH, H., 1938. Experience and Prediction. (The University of Chicago Press: Chicago).

ROLLER, D. y ROLLER, D.H.D., 1954. The Development of the Concept of Electric Change. Electricity from the Greeks to Coulomb. En Harvard Case Histories in Experimental Science. J.B., Conant (Ed.). (Harvard University Press: Cambridge, Mass), 3 reimpresión 1970.

SCHWAB, J., 1964. Problems, Topics and Issues. En Education and the Structure of Knowledge. (Rand McNally: Chicago).

STEIN, B. y BRANSFORD, I.D., 1979. Constraints on Effective Elaboration: Effects of Precision and Subject Generation. Journal of Verbal Learning and Verbal Behavior, 18 , pp. $769-777$.

THOMSON, W., 1882. On the dynamical theory of heat, with numerical results deduced from Mr. Joule's equivalent of a thermal unit, and M. Regnault's observations on steam. En Mathematical and Physical Papers of William Thomson. Vol. 1. (Cambridge University Press: Cambridge).

TIPLER, P.A., 1976. Physics. (Worth Pub: Nueva York), (Traducción castellāna. Ed. Reverté, 1985).

TOULMIN, S., 1972. Human Understanding. (Princeton University Press: Princenton). (Taducción castellana. Alianza Editorial, 1978). 